\title{
An eight-year study of Shigella species in Beijing, China: serodiversity, virulence genes, and antimicrobial resistance
}

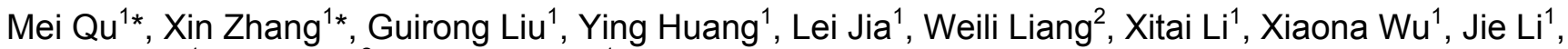 \\ Hanqiu Yan ${ }^{1}$, Biao Kan², Quanyi Wang ${ }^{1}$ \\ * Authors contributed equally to this work. \\ ${ }^{1}$ Beijing Key Laboratory of Diagnostic and Traceability Technologies for Food Poisoning, Beijing Centre for Disease \\ Prevention and Control, Capital Medical University School of Public Health and Family Medicine, Beijing, China \\ ${ }^{2}$ State Key Laboratory for Infectious Disease Prevention and Control, National Institute for Communicable Disease \\ Control and Prevention, Beijing, China
}

\begin{abstract}
Introduction: This study was conducted to determine the prevalence of serotypes, virulence factors, and antimicrobial resistance patterns of Shigella spp. in Beijing, China, from 2004 to 2011.

Methodology: Real-time PCR assays were used to detect virulent genes, and the Kirby-Bauer disk diffusion method was used to evaluate antimicrobial resistance.

Results: Among the total of 1,652 Shigella isolates, S. sonnei (57.1\%) was the predominant species, followed by $S$. flexneri (42.3\%), S. dysenteriae $(0.4 \%)$, and $S$. boydii $(0.2 \%)$. Nineteen serotypes were discovered among $S$. flexneri strains. The virulence gene ipa $\mathrm{H}$ was the most frequent, followed by sen and set. The presence of set showed significant difference in two dominant serogroups, S. flexneri and $S$. sonnei. Over $90 \%$ of Shigella isolates showed resistance to at least three drugs with widened spectrum. High-level antimicrobial resistance to single and multiple antibiotics was more common among $S$. sonnei than $S$. flexneri.

Conclusion: There was an obvious serotype change and a dramatic increase of antibiotic resistance in Shigella prevalence in Beijing.
\end{abstract}

Key words: Shigella; serodiversity; prevalence; antimicrobial resistance; diarrhea.

J Infect Dev Ctries 2014; 8(7):904-908. doi:10.3855/jidc.3692

(Received 19 April 2013 - Accepted 29 May 2013)

Copyright $(92014 \mathrm{Qu}$ et al. This is an open-access article distributed under the Creative Commons Attribution License, which permits unrestricted use, distribution, and reproduction in any medium, provided the original work is properly cited.

\section{Introduction}

Shigellosis, as a major source of diarrhea, is a global human health problem. Any of four species of Shigella ( $S$. dysenteriae, S. flexneri, S. boydii, and $S$. sonnei) can cause shigellosis. S. sonnei and $S$. boydii, associated with mild illness of short duration, most often occur in developed countries; S. flexneri is the most prevalent species in developing countries, and $S$. dysenteriae is known to cause sporadic outbreaks and epidemics worldwide, with severe complications and very high mortality [1-3]. In China, Shigella spp. is the most frequently isolated diarrheal pathogen, accounting for up to 1.7 million episodes of bacillary dysentery annually, with an estimated 200,000 patients admitted to hospitals [4].

Virulence genes responsible for the pathogenesis of shigellosis are often multifactorial and coordinately regulated. IpaH is present in multiple copies on both the invasion plasmid and on the chromosome, and is responsible for the modification of host response to infection. Another two genes encoding enterotoxins, set and sen, have roles in altering electrolyte and water transport in the small intestine during the initial watery phase of shigellosis [5,6]. Knowledge about the distribution of the three genes is limited, especially for the last two, which has been reported only in $S$. flexneri serotypes in some detail $[7,8]$. The purpose of this study is to describe the prevalence of various Shigella species, the distribution of three virulence genes, and the local antibiotic resistance patterns in Beijing, the capital of China, between 2004 and 2011.

\section{Methodology}

Shigella isolates and bacteriological examination

A hospital-based active surveillance was conducted in 16 districts of Beijing from 2004 to 2011. The sentinel hospitals collected stool specimens of outpatients, with a monthly enrollment number of 20 
30 patients per district. For isolation of Shigella, the fecal swabs were plated directly onto SalmonellaShigella agar and incubated at $37^{\circ} \mathrm{C}$ for 16 to 24 hours. After culture and screening, the biochemical identification was confirmed with the VITEK 2 Compact instrument (bioMérieux, Marcy l'Etoile, France). Finally, serologic identification was performed by the slide agglutination test.

\section{Real-time PCR assay}

Real-time PCR assays were employed to detect for the presence of $i p a \mathrm{H}$, set and sen genes. For $i p a \mathrm{H}$, ipa $\mathrm{H}-\mathrm{P}$ (FAMCTGTCGAAGCTCCGCAGAGGCAC-TAMRA), ipa H-F (5'-CATCAGCAGCAACAGTGAAAGAC3') and ipaH-R (5'-CACGCAATACCTCCGGATTC3') were used; for set, set-P (FAMCCCGGCACCTGTGGAATGGC-TAMRA), set-F (5'-GGTTCACGCTACCATCAAAGATTAC-3'), and set-R (5'-GCTGACCGGGAATATGGATGT-3') were used; for sen, sen-P (FAMCGTCCTCCCATTCCTTCCGCAG-TAMRA), sen-F (5'- AGTGCTTGGGATAAACCCGATA-3'), and sen-R (5'-ACGGAGAACTCTTGGAAACTTCTG-3') were used. The real-time PCR assay was performed using the TaqMan Universal PCR Master Mix Kit (Applied Biosystems, Foster City, United States).

\section{Antimicrobial resistance testing}

Antibiotic resistance of the Shigella isolates was tested using the Kirby-Bauer disk diffusion method, following the guidelines of the Clinical and Laboratory Standards Institute (CLSI 2005). Escherichia coli (ATCC 25922) was included in the test as a quality control. The disk concentrations of 12 antimicrobial agents (Oxoid, Basingstoke, United Kingdom) were as follows: $10 \mu \mathrm{g}$ ampicillin (AMP), 5 $\mu \mathrm{g} \quad$ ciprofloxacin (CIP), $25 \quad \mu \mathrm{g}$ sulphamethoxazole/trimethoprim $\quad(\mathrm{SXT}), \quad 30 \mu \mathrm{g}$ nalidixic acid (NAL), $30 \mu \mathrm{g}$ tetracycline (TET), $30 \mu \mathrm{g}$ amoxicillin clavulanic acid (AMC), $10 \mu \mathrm{g}$ gentamicin (GEN), $10 \mu \mathrm{g}$ norfloxacin (NOR), $5 \mu \mathrm{g}$ ofloxacin (OFX), $30 \mu \mathrm{g}$ cefazolin (KZ), $30 \mu \mathrm{g}$ cephalothin (KF), and $30 \mu \mathrm{g}$ cefotaxime (CTX). Multidrug resistance was defined as resistance to at least three classes of antibiotics.

\section{Statistical analysis}

Statistical analysis was performed with SPSS version 11.5 software. Comparison of proportions and statistical significance were calculated using the twotailed Chi-square test.

\section{Results}

During the eight-year study period, a total of 1,652 isolates received were confirmed as Shigella and serotyped. The species distribution over time is shown in Figure 1. S. sonnei was the most prevalent serogroup ( $\mathrm{n}=943,57.1 \%)$, followed by $S$. flexneri $(\mathrm{n}$ $=698,42.3 \%), S$. dysenteriae $(\mathrm{n}=7,0.4 \%)$, and $S$. boydii $(\mathrm{n}=4,0.2 \%)$. The highest number of infections were recorded in 2010, with 322 isolations, whereas the number of isolations decreased to below 300 in other years, with obvious fluctuations. S. flexneri was the most common species isolated in $2004(69.4 \%)$, 2005 (73.8\%), 2006 (60.7\%), and 2008 (50.7\%). In 2007, a shift occurred where S. sonnei (57.9\%) replaced $S$. flexneri $(40.4 \%)$ as the most prevalent species. Subsequently, the relative importance of $S$. sonnei gradually increased, and it accounted for $85.0 \%$ of the dominant species instead of S. flexneri (14.5\%) in 2011.

The trends in 19 serotypes of $S$. flexneri are described in Figure 2. The seven most frequently isolated serotypes (in order of prevalence: 4c, 2a, 1a, 2 b, 5 b, var x 4 ) were responsible for $94.1 \%$ of all $S$. flexneri episodes, whereas any other serotype was

Figure 1. Distribution of Shigella species from 2004 to 2011 in Beijing

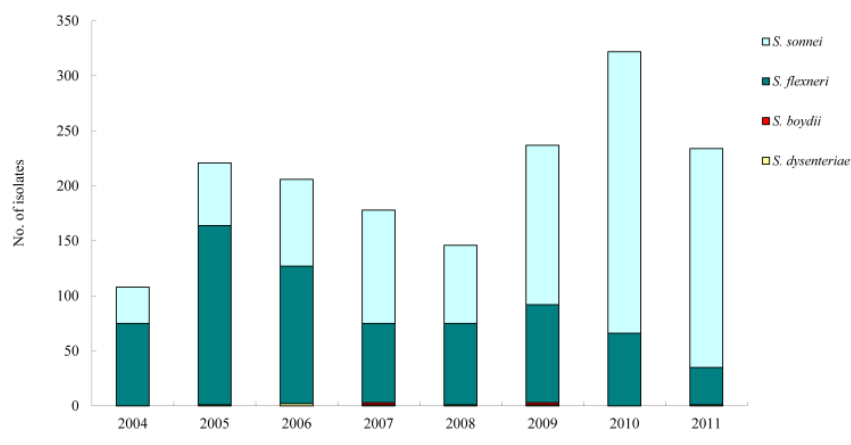

Figure 2. Distribution of Shigella flexneri serotype from 2004 to 2011 in Beijing

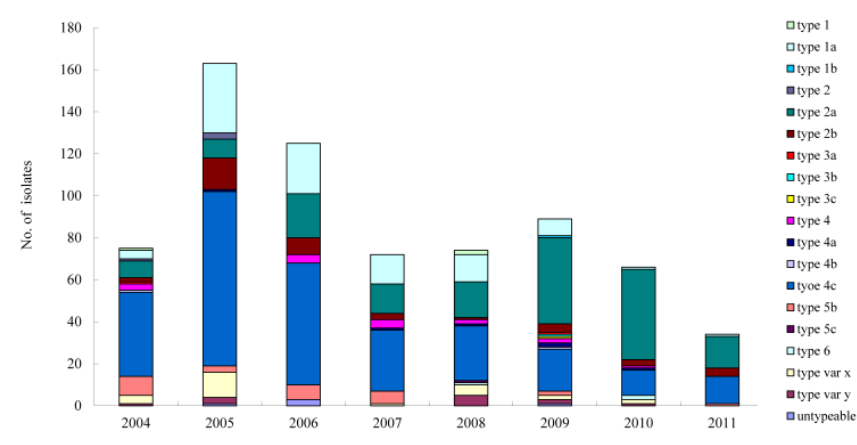


present in less than $1.0 \%$. For three major serotypes $4 \mathrm{c}, 1 \mathrm{a}$, and $2 \mathrm{a}$ - the former two showed a pronounced decrease with occasional inter-annual variations, while the third had an obvious fluctuation over time, with peaks in 2009 and 2010. The other infrequently observed serotypes, such as $2 \mathrm{~b}, 4,5 \mathrm{~b}$, var $\mathrm{x}$ and so on, varied in different years. Statistically significant shifts in the relative proportions of $S$. flexneri serotypes were observed during the period.

The detection of the virulence genes from 274 Shigella strains revealed that ipa $\mathrm{H}(96.3 \%)$ was the most frequent, followed by sen $(85.8 \%)$ and set (46.0\%) (Supplementary Table 1). Of note, all the strains positive for set were observed with the presentence of both ipa $\mathrm{H}$ and sen. Of the 274 strains, $43.1 \%$ were found to be positive for $i p a \mathrm{H}+$ set + sen + , $85.8 \%$ for $i p a \mathrm{H}+$ sen,$+ 46.0 \%$ for $i p a \mathrm{H}+$ set + , and $43.1 \%$ for set + sent. The prevalence of the three virulence genes among diverse Shigella spp. was analyzed. The ipa $\mathrm{H}$ gene was detected in $97.0 \%$ of $135 \mathrm{~S}$. flexneri isolates, $99.2 \%$ of $133 \mathrm{~S}$. sonnei isolates, one of four $S$. boydii isolates, and none of two $S$. dysenteriae isolates. The set gene was present in $88.1 \%$ of $S$. flexneri isolates and in $5.3 \%$ of $S$. sonnei isolates, whereas it was absent in $S$. boydii and $S$. dysenteriae isolates. The sen gene was present in $88.9 \%$ of $S$. flexneri isolates, $85.7 \%$ of $S$. sonnei isolates, one $S$. boydii isolate, and none of the $S$. dysenteriae isolates. The set gene showed the difference of distribution in two dominant serogroups, S. flexneri $(88.1 \%)$ and S. sonne $(5.3 \%)(\mathrm{p}<0.001)$. As for ipa $\mathrm{H}$ and sen, there were no statistically significant differences in different Shigella species $(\mathrm{p}$ $>0.05)$.

Antimicrobial drug testing was carried out on 872 Shigella isolates, including $495 \mathrm{~S}$. sonnei, $369 \mathrm{~S}$. flexneri, as well as three $S$. dysenteriae and three $S$. boydii isolates (Supplementary Table 2). Resistance to one or more drugs was observed in $98.9 \%$ of the 872 isolates. The highest resistance was detected for NAL (90.7\%), followed by TET (89.9\%), AMP (86.9\%), and SXT (81.5\%). Moderate resistance of Shigella isolates to GEN (49.2\%) also was detected; however, resistance to NOR or OFX was relatively low $(9.9 \%$ and $9.7 \%$, respectively).

The resistance to individual antimicrobials varied by species. The majority of $S$. sonnei isolates were resistant to NAL (96.4\%), SXT (93.8\%), or TET (93.4\%), whereas the resistance was lower among $S$. flexneri isolates $(83.7 \%, 65.9 \%$, and $85.9 \%$, respectively). Furthermore, the differences of resistance to these agents was statistically significant $(\mathrm{p}<0.001)$. For GEN, $78.5 \%$ of $S$. flexneri had high level of resistance, as did only $10.3 \%$ of $S$. sonnei isolates. S. flexneri showed more resistance to AMC, CIP, NOR, and OFX compared to S. sonnei $(18.4 \%-$ $40.7 \%$ versus $3.0 \%-8.0 \%, \mathrm{p}<0.001)$, but $S$. sonnei tended to demonstrate an intermediate level of resistance to $\mathrm{KF}, \mathrm{KZ}$, and CTX, higher than $S$. flexneri did $(22.7 \%-46.5 \%$ versus $10.8 \%-16.3 \%, \mathrm{p}<0.001)$ (Supplementary Table 2).

In addition, multidrug resistance (MDR) is also important to consider. In this study, $90.8 \%$ of Shigella isolates were resistant to at least three antimicrobial drug subclasses, $82.2 \%$ were resistant to four, $70.4 \%$ were resistant to five, and $39.7 \%$ were resistant to six. Among many different patterns, the five most predominant resistance patterns were AMP/NAL/TET (78.4\%), NAL/SXT/TET (76.4\%), AMP/SXT/TET (72.2\%), AMP/NAL/SXT (71.6\%), and AMP/NAL/SXT/TET (70.5\%) (Supplementary Table 3). There were also notable differences in MDR phenotypes between $S$. sonnei and $S$. flexneri. The dominant pattern AMP/NAL/TET was more common among $S$. sonnei than among $S$. flexneri $(83.1 \%$ versus $72.4 \%, \mathrm{p}<0.001)$. For $S$. sonnei, the most common phenotype was NAL/SXT/TET, which was much higher than for S. flexneri $(90.5 \%$ versus $58.3 \%$, p < $0.001)$.

\section{Discussion}

Shigellosis is the most important cause of diarrhea worldwide, especially in underdeveloped and developing nations with substandard hygiene and poor quality water supplies. In this study, we found an unexpectedly complex landscape of circulating Shigella strains in Beijing. S. flexneri was the most common of the four species from 2004 to 2006, whereas $S$. sonnei has replaced $S$. flexneri as the most prevalent serogroup since 2009. These findings differ from those of studies conducted in developing countries [9] and other provinces in China [10], where $S$. flexneri was still the most frequently isolated species; however, our results match the findings in developed countries [2,11] and Thailand [12], which is rapidly becoming industrialized. The reason for this change is not known, but improvement of environmental conditions, hygiene habits, and water supplies should be considered. The significant increase in percentage of $S$. sonnei accompanied by the obvious reduction in S. flexneri may reflect that Beijing has undergone considerable socioeconomic changes during the period, with the infection pattern having 
changed from that of a developing city to that of a developed city.

Amongst the $S$. flexneri isolates, there were a surprisingly wide range of 19 serotypes, with $4 \mathrm{c}$ and $2 \mathrm{a}$ being the most prevalent serotypes. S. flexneri $2 \mathrm{a}$ is still the most common in many developing countries, including China $[1,4,12]$. It should be noted that $S$. flexneri serotype $4 \mathrm{c}$ has remained in continuous existence over time in Beijing, although it has rarely been reported in other studies. We found statistically significant shifts in $S$. flexneri serotypes between observation years, consistent with previous works in India and Chile [13,14]. The variety in Shigella species and serotypes shows high heterogeneous characteristics in temporal distribution, which further emphasizes the importance of identifying isolates to serotype level for the implementation of effective control strategies.

In the present study, the prevalence of ipa $\mathrm{H}$ was independent of the four different species of Shigella tested, confirming previous works that the gene is highly conserved in various serotypes [9]. The set and sen genes were found to be widely distributed in various $S$. flexneri serotypes, unlike in previous studies where they were almost exclusively found in $S$. flexneri $2 \mathrm{a}$ isolates and rarely in other species or serotypes [5-8,15]. Overall, the present study detected $i p a \mathrm{H}$, set, and sen in all the prevalent serotypes of $S$. flexneri; however, the presentence of set in S. sonnei was much lower than in sen and ipa $\mathrm{H}$. Working on temporal variations of diverse species, our results enhance knowledge on the prevalence and distribution of these genes, which might assist in developing markers for Shigella of different types.

This study demonstrated the drastically increasing spectrum of antimicrobial resistance in Shigella isolates. All Shigella species also exhibited a very high rate of resistance $(80 \%-90 \%)$ to older generation antimicrobials such as NAL, TET, AMP, and SXT. Although a similar pattern of resistance has been reported in our region and in other Asian countries $[4,16,17]$, the frequency of resistance reported here is higher than frequencies observed earlier, and the trend to MDR is more pronounced [3,4]. Cephalosporins and fluoroquinolones were two popular empirical options to treat severe gastrointestinal infections caused by pathogenic bacteria. Our finding of a $17.8 \%$ resistance rate to the third-generation cephalosporins (CTX) was in agreement with observations from India and Cameroon, where resistance rates of $12 \%$ and $20 \%$, respectively, were reported $[16,18]$. Additionally, our study found a $10 \%$ resistance rate to other fluoroquinolones such as CIP, NOR, and OFX. It has been noted that in other countries with strict antimicrobial controls (e.g., the United States), less than $1 \%$ of Shigella isolates were resistant to cephalosporins and fluoroquinolones [19]. In China, the use of antimicrobial drugs has been poorly regulated and the indiscriminate overuse of antibiotics remains a serious issue that might be responsible for the increase of multiple antimicrobial resistance at an alarming speed.

In the present study, we found that $S$. sonnei was more frequently resistant to NAL, TET, and SXT, alone or in combination, than was $S$. flexneri. Other studies showed that $S$. sonnei had lower rates of resistance, compared with $S$. flexneri, to most of the antibiotics [19]. A possible explanation is that infections due to $S$. sonnei are becoming more and more common than infections due to the other Shigella species in Beijing, making exposure to selective pressure from antibiotics more likely.

In summary, this study provides initial data on the prevalence and distribution of ipa $\mathrm{H}$, set, and sen genes in a wide variety of Shigella isolates over an eightyear period. S. sonnei has replaced $S$. flexneri as a dominant species with high rates of multidrug resistances in Beijing. Continuous local monitoring of resistance patterns is necessary for effective therapy and control measures against shigellosis.

\section{Acknowledgements}

This work was supported by a grant from the National Key Program for Infectious Disease of China (2012ZX10004215-003001) and the Project of Beijing High-Level Technical Personnel Training in Health (2013-3-099).

\section{References}

1. Kotloff KL, Winickoff JP, Ivanoff B, Clemens JD, Swerdlow DL, Sansonetti PJ, Adak GK, Levine MM (1999) Global burden of Shigella infections: implications for vaccine development and implementation of control strategies. Bull World Health Organ 77: 651-666.

2. Gupta A, Polyak CS, Bishop RD, Sobel J, Mintz ED (2004) Laboratory-confirmed shigellosis in the United States, 19892002: epidemiologic trends and patterns. Clin Infect Dis 38: 1372-1377.

3. Toro CS, Farfan M, Contreras I, Flores O, Navarro N, Mora GC, Prado V (2005) Genetic analysis of antibiotic-resistance determinants in multidrug-resistant Shigella strains isolated from Chilean children. Epidemiol Infect 133: 81-86.

4. Wang XY, Tao F, Xiao D, Lee H, Deen J, Gong J, Zhao Y, Zhou W, Li W, Shen B (2006) Trend and disease burden of bacillary dysentery in China (1991-2000). Bull World Health Organ 84: 561-568.

5. Noriega FR, Liao FM, Formal SB, Fasano A, Levine MM (1995) Prevalence of Shigella enterotoxin 1 among Shigella 
clinical isolates of diverse serotypes. J Infect Dis 172: 14081410 .

6. Vargas M, Gascon J, Jimenez De Anta MT, Vila J (1999) Prevalence of Shigella enterotoxins 1 and 2 among Shigella strains isolated from patients with traveler's diarrhea. J Clin Microbiol 37: 3608-3611.

7. Niyogi SK, Vargas M, Vila J (2004) Prevalence of the sat, set and sen genes among diverse serotypes of Shigella flexneri strains isolated from patients with acute diarrhoea. Clin Microbiol Infect 10: 574-576.

8. Yavzori M, Cohen D, Orr N (2002) Prevalence of the genes for shigella enterotoxins 1 and 2 among clinical isolates of shigella in Israel. Epidemiol Infect 128: 533-535.

9. Faruque SM, Khan R, Kamruzzaman M, Yamasaki S, Ahmad QS, Azim T, Nair GB, Takeda Y, Sack DA (2002) Isolation of Shigella dysenteriae type 1 and S. flexneri strains from surface waters in Bangladesh: comparative molecular analysis of environmental Shigella isolates versus clinical strains. Appl Environ Microbiol 68: 3908-3913.

10. Xia S, Xu B, Huang L, Zhao JY, Ran L, Zhang J, Chen H, Pulsrikarn C, Pornruangwong S, Aarestrup FM (2011) Prevalence and characterization of human Shigella infections in Henan Province, China, in 2006. J Clin Microbiol 49: 232242.

11. Ekdahl K, Andersson Y (2005) The epidemiology of travelassociated shigellosis--regional risks, seasonality and serogroups. J Infect 51: 222-229.

12. Bangtrakulnonth A, Vieira AR, Lo Fo Wong DM, Pornreongwong S, Pulsrikarn C, Sawanpanyalert P, Hendriksen RS, Aarestrup FM (2008) Shigella from humans in Thailand during 1993 to 2006: spatial-time trends in species and serotype distribution. Foodborne Pathog Dis 5: 773-784.

13. Dutta S, Rajendran K, Roy S, Chatterjee A, Dutta P, Nair GB, Bhattacharya SK, Yoshida SI (2002) Shifting serotypes, plasmid profile analysis and antimicrobial resistance pattern of shigellae strains isolated from Kolkata, India during 19952000. Epidemiol Infect 129: 235-243.
14. Fulla N, Prado V, Duran C, Lagos R, Levine MM (2005) Surveillance for antimicrobial resistance profiles among Shigella species isolated from a semirural community in the northern administrative area of santiago, chile. Am J Trop Med Hyg 72: 851-854.

15. Roy S, Thanasekaran K, Dutta Roy AR, Sehgal SC (2006) Distribution of Shigella enterotoxin genes and secreted autotransporter toxin gene among diverse species and serotypes of shigella isolated from Andaman Islands, India. Trop Med Int Health 11: 1694-1698.

16. Tiruneh M (2009) Serodiversity and antimicrobial resistance pattern of Shigella isolates at Gondar University teaching hospital, Northwest Ethiopia. Jpn J Infect Dis 62: 93-97.

17. Bhattacharya D, Sugunan AP, Bhattacharjee H, Thamizhmani R, Sayi DS, Thanasekaran K, Manimunda SP, Ghosh AR, Bharadwaj AP, Singhania M (2012) Antimicrobial resistance in Shigella--rapid increase \& widening of spectrum in Andaman Islands, India. Indian J Med Res 135: 365-370.

18. Njunda AL, Assob JC, Nsagha DS, Kamga HL, Awafong MP, Weledji EP (2012) Epidemiological, clinical features and susceptibility pattern of shigellosis in the buea health district, Cameroon. BMC Res Notes 5: 54.

19. Shiferaw B, Solghan S, Palmer A, Joyce K, Barzilay EJ, Krueger A, Cieslak P (2012) Antimicrobial susceptibility patterns of Shigella isolates in Foodborne Diseases Active Surveillance Network (FoodNet) sites, 2000-2010. Clin Infect Dis 54 Suppl 5: S458-S463.

\section{Corresponding author}

Quanyi Wang

Department of Infectious and Endemic Diseases Control

Beijing Centre for Disease Prevention and Control

No. 16, He Ping Li Middle Street, Beijing, 100013, China

Phone: +86-10-64407109

Fax: +86-10-64407113

Email: bjcdcxm@126.com

Conflict of interests: No conflict of interests is declared. 


\section{Supplementary Items}

Supplementary Table 1. Prevalence of three virulence genes in Shigella strains by species

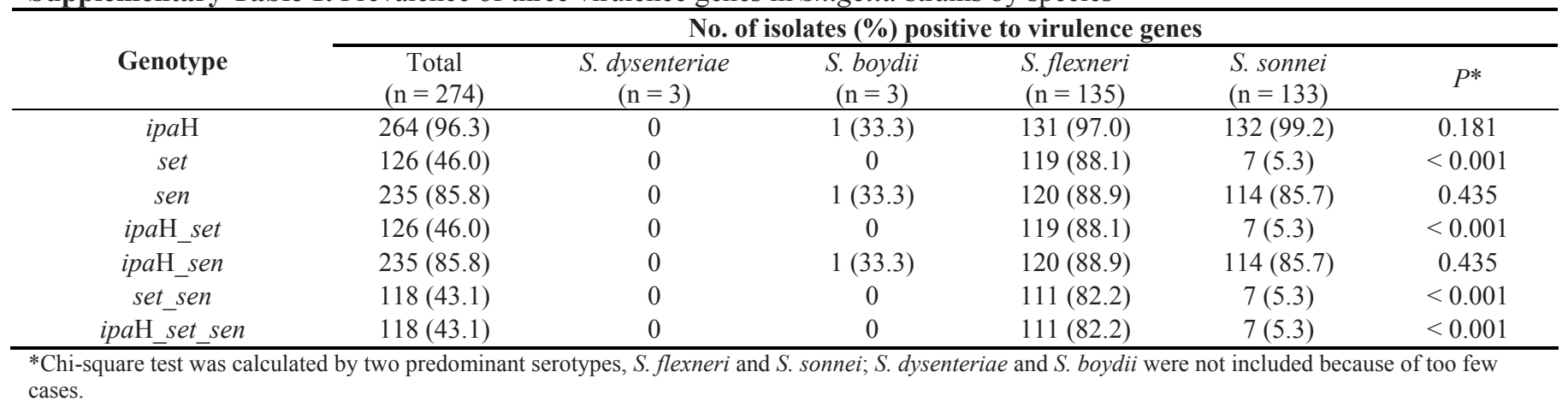

Supplementary Table 2. Antimicrobial resistance of Shigella strains by species

\begin{tabular}{|c|c|c|c|c|c|c|}
\hline \multirow[b]{2}{*}{ Antimicrobial agent } & \multicolumn{6}{|c|}{ No. of isolates $(\%)$ resistant to various antimicrobial agent } \\
\hline & $\begin{array}{c}\text { Total } \\
(\mathrm{n}=872)\end{array}$ & $\begin{array}{c}\text { S. dysenteriae } \\
(\mathrm{n}=3)\end{array}$ & $\begin{array}{c}\text { S. boydii } \\
(\mathrm{n}=3)\end{array}$ & $\begin{array}{l}\text { S. flexneri } \\
(\mathrm{n}=369)\end{array}$ & $\begin{array}{l}\text { S. sonnei } \\
(\mathrm{n}=497)\end{array}$ & $P^{*}$ \\
\hline NAL & $791(90.7)$ & $2(66.6)$ & $1(33.3)$ & $309(83.7)$ & $479(96.4)$ & $<0.001$ \\
\hline AMP & $758(86.9)$ & $2(66.6)$ & $1(33.3)$ & $324(87.8)$ & $431(86.7)$ & 0.637 \\
\hline SXT & $711(81.5)$ & $1(33.3)$ & $1(33.3)$ & $243(65.9)$ & $466(93.8)$ & $<0.001$ \\
\hline GEN & $429(49.2)$ & 0 & $1(33.3)$ & $38(10.3)$ & $390(78.5)$ & $<0.001$ \\
\hline $\mathrm{KZ}$ & $203(23.3)$ & $2(66.6)$ & $1(33.3)$ & $49(13.3)$ & $151(30.4)$ & $<0.001$ \\
\hline $\mathrm{AMC}$ & $190(21.8)$ & 0 & 0 & $150(40.7)$ & $40(8.0)$ & $<0.001$ \\
\hline CTX & $155(17.8)$ & $1(33.3)$ & $1(33.3)$ & $40(10.8)$ & $113(22.7)$ & $<0.001$ \\
\hline CIP & $93(10.7)$ & $2(66.6)$ & 0 & $82(22.2)$ & $9(1.8)$ & $<0.001$ \\
\hline NOR & $86(9.9)$ & $2(66.6)$ & 0 & $75(20.3)$ & $9(1.8)$ & $<0.001$ \\
\hline OFX & $85(9.7)$ & 2 (66.6) & 0 & $68(18.4)$ & $15(3.0)$ & $<0.001$ \\
\hline
\end{tabular}

*Chi-square test was calculated by two predominant serotypes, S. flexneri and S. sonnei; S. dysenteriae and S. boydii were not included because of too few cases.

Supplementary Table 3. Predominant multidrug resistance patterns of Shigella strains by species

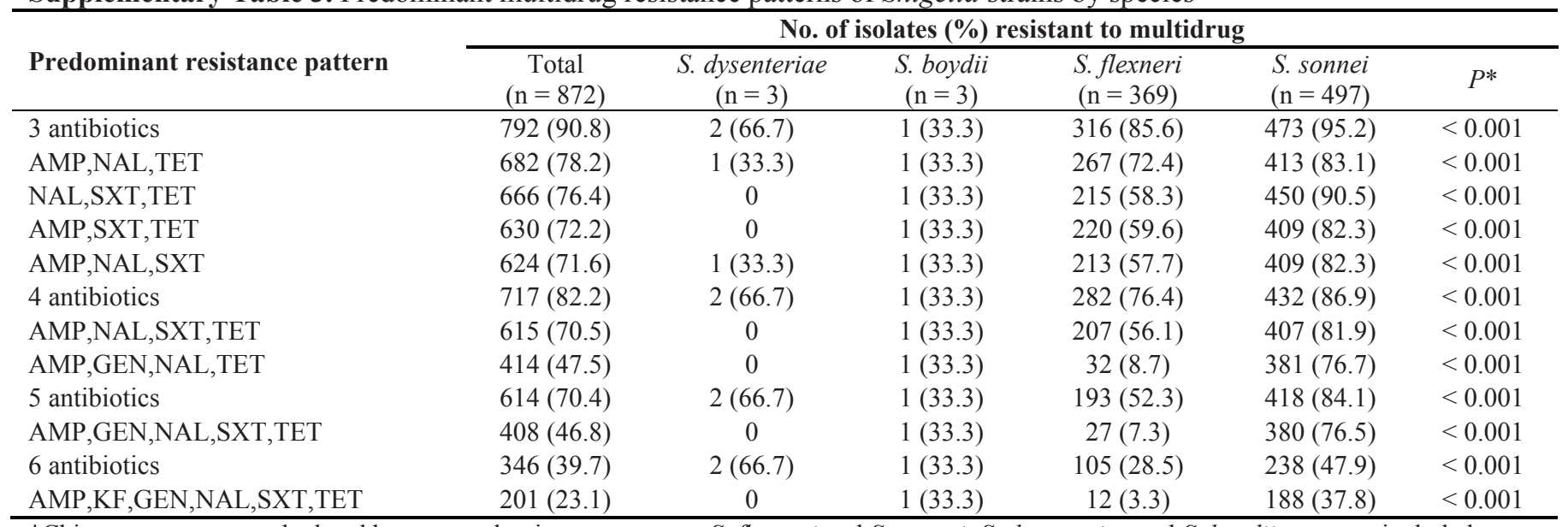

*Chi-square test was calculated by two predominant serotypes, S. flexneri and S. sonnei; S. dysenteriae and S. boydii were not included because of too few cases. 